bacteria. Further, bacteria vary in their copy number of the $16 \mathrm{~S}$ rRNA gene, dependent on species. Despite these limitations, our associations have reached a strong level of significance.

\section{S114 PREVIOUSLY UNRECOGNISED ORAL ANAEROBES IN PLEURAL INFECTION}

JM Wrightson, JA Wray, TL Street, SJ Chapman, DWM Crook, NM Rahman. University of Oxford, Oxford, UK

\subsection{6/thoraxjnl-2014-206260.120}

Laboratory culture of pleural infection samples is positive in only $30 \%$ of cases, probably related to antibiotic usage and fastidious or unculturable organisms such as some anaerobes. Previous studies using capillary sequencing of the $16 \mathrm{~S}$ rRNA gene improves rates of organism identification, but is unable to resolve the polymicrobiality thought to be present in anaerobic infection.

We used ultra-deep pyrosequencing to definitively characterise anaerobic pleural infection.

Methods Pleural infection samples were obtained from the Second Multicentre Intrapleural Sepsis randomised controlled Trial (MIST2), REC no. 04/MRE5/53. DNA was extracted using the FastDNA SPIN Kit. Modified 'fusion' primers amplified the V46 regions of the $16 \mathrm{~S}$ rRNA gene. Subsequent pyrosequencing was performed on the Roche 454 GS FLX instrument. Data analyses were performed using the open souce 'Quantitative Insights Into Microbial Ecology' platform. Strategies were used to control for contamination.

Results 172 pleural fluid samples were available, 98 of which were successfully sequenced. 32/98 samples contained anaerobes (defined when $\geq 10 \%$ of sequences in a sample represented anaerobes).

Fusobacteriales, particularly Fusobacterium nucleatum, and Bacteroidales, particularly Prevotella spp. were commonly found although other anaerobes were seen (see Figure).

Anaerobic pleural infection was usually polymicrobial, with an estimated 4-5 operational taxonomic units ("species") per sample. Particular patterns of co-infection were Fusobacterium nucelatum and Streptococcus 'milleri' group although Prevotella spp. \pm Fusobacterium spp. \pm Porphyromonas spp. \pm Treponema spp. also co-infected several samples.

Many species were found that have not been previously documented, including Atopobium rimae, Cryptobacterium curtum, Lactobacillus spp., Stomatobaculum spp., Oribacterium spp., Prevotella baroniae, Prevotella dentalis/Hallella seregens, Prevotella scopos, Fretibacterium spp., Tanerella forsythia, Treponema denticola, lecithinolyticum, maltophilum, medium and socranskii. Intriguingly, the original isolation and description of almost all these anaerobes were from the oropharynx and some have never been detected at other body sites.



Anaerobic Gram negative bacilli

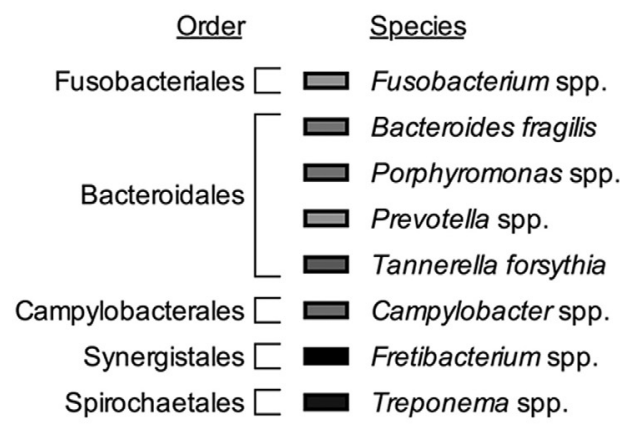

Anaerobic Gram positive cocci Clostridiales $\sqsubset \square$ Panimonas micra

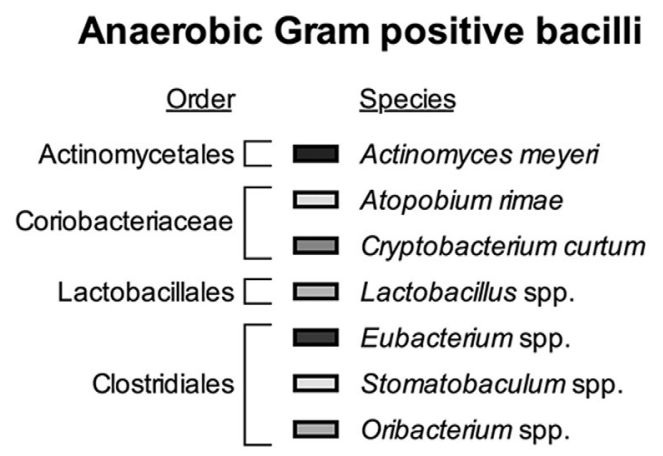

\section{Other}

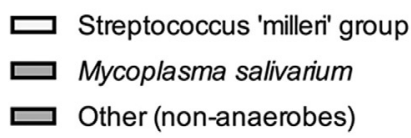

Abstract S114 Figure 1 Individual bacteriology of all samples that had anaerobes account for at least $10 \%$ of sequence reads 
Conclusions Anaerobic infection occurs in $\sim 33 \%$ of cases of pleural infection and is typically polymicrobial. Sequencing revealed many anaerobic bacteria never previously isolated in the pleural space. These bacteria have a strong association with the oropharynx, particularly the gingival crevices. Such findings add to our understanding of the mechanism of development of pleural infection.

\section{S115 PLEURAL FLUID ADENOSINE DEAMINASE (ADA) IN THE DIAGNOSIS OF TUBERCULOUS PLEURAL EFFUSIONS IN A LOW INCIDENCE POPULATION}

${ }^{1} \mathrm{D}$ Arnold, ${ }^{1} \mathrm{R}$ Bhatnagar, ${ }^{2} \mathrm{~L}$ Fairbanks, ${ }^{1} \mathrm{~N}$ Zahan-Evans, ${ }^{1} \mathrm{~A}$ Clive, ${ }^{1} \mathrm{~A}$ Medford, ${ }^{1} \mathrm{~N}$ Maskell. ${ }^{1}$ Academic Respiratory Unit, Bristol, UK; ${ }^{2}$ Guy's and St Thomas' Hospital, London, UK

\subsection{6/thoraxinl-2014-206260.121}

Introduction Numerous studies have assessed the diagnostic ability of pleural adenosine deaminase (ADA) in detecting tuberculous pleural effusions, with good specificity and sensitivity reported. However, in the UK (UK) ADA is not routinely used in the investigation of a patient with a pleural effusion, mainly due to a lack of evidence as to its utility in areas where tuberculosis (TB) incidence is low.

Methods Patients presenting with an undiagnosed pleural effusion to a tertiary pleural centre in South-West England over a 3 year period, were prospectively recruited to a pleural biomarker study, in which baseline pleural fluid samples were collected and stored. Samples from consecutive patients with robust 12-month follow up data and confirmed diagnoses were sent for ADA analysis.

Results Of 338 patients enrolled, 7 had confirmed tuberculous pleural effusion (2\%). All 7 TB effusions were lymphocyte predominant with a median ADA of $72.0 \mathrm{IU} / \mathrm{L}$ (range- 26.7 to 91.5 ) compared to a population median of $12.0 \mathrm{IU} / \mathrm{L}$ (range- 0.3 to 568.4). Using the established cut off of $35 \mathrm{IU} / \mathrm{L}$, ADA was shown to have a negative predictive value (NPV) of 99.7\% (95\% CI; 98.2-99.9\%) for the exclusion of TB, and sensitivity of $85.7 \%$ (95\% CI; 42.2-97.6\%) with an area under the curve of $0.88(95 \%$ CI; 0.732-1.000). In the context of a lymphocytic effusion an ADA over $35 \mathrm{IU} / \mathrm{L}$ had a sensitivity and positive predictive value of 85.7\% (95\% CI; 42.2-97.6\%), see figure. Bacterial pleural infection was the main alternative cause of raised ADA in our cohort.

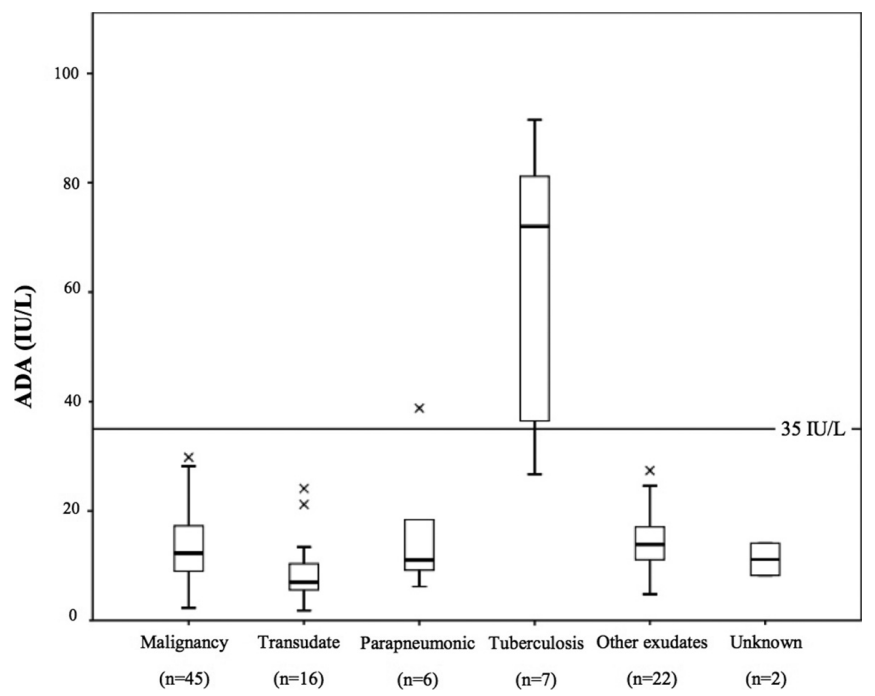

Abstract S115 Figure 1 Boxplot of ADA levels by diagnostic category in lymphocyte predominant effusions
Discussion This is the first study examining the diagnostic utility of pleural fluid ADA in a low TB incidence area. The chance of an effusion with an ADA under $35 \mathrm{IU} / \mathrm{L}$ being of tuberculous aetiology was negligible and empirical anti-TB therapy could be avoided in such cases. A pleural ADA of over 35 IU/L in lymphocyte-predominant pleural fluid gives a strong suspicion of tuberculous aetiology. In patients who are unsuitable for more invasive procedures this could be used as an indication to start therapy.

\section{S116 SYSTEMIC CHEMOTHERAPY AND THE RISK OF PLEURAL INFECTION WITH INDWELLING PLEURAL CATHETERS (IPCS)}

SC Gunatilake, R Mercer, S Babu, L Bishop, AJ Chauhan. Portsmouth Hospitals NHS Trust, Portsmouth, UK

10.1136/thoraxjnl-2014-206260.122

Introduction Indwelling pleural catheters (IPCs) are an alternative method for controlling recurrent, symptomatic malignant pleural effusions, currently reserved for cases of known trapped lung, or after an initial unsuccessful talc pleurodesis. ${ }^{1}$ A $2.8 \%$ pleural infection rate has been observed in patients with an IPC ${ }^{2}$ leading to the concern that this risk of infection may increase further with systemic chemotherapy.

Objectives

- Determine whether systemic chemotherapy increased the number of IPC related pleural infections.

- Whether the risk was related to the interval between chemotherapy and IPC insertion.

Methods A detailed retrospective review was performed of the first 100 patients treated with an IPC from February 2011 to December 2013 at Queen Alexandra Hospital, Portsmouth.

Results 11 of the 100 IPC insertions developed pleural infection (11\%), four of whom had received chemotherapy (see table). Overall 15 patients received chemotherapy after IPC insertion with an interval varying from 0 days (same day) to 43 days, with a median of 9 days. The $2 \times 2$ table compared the proportion of patients developing pleural infection with and without chemotherapy.

Further analyses assessed the interval from chemotherapy to IPC insertion and whether it influenced the numbers of pleural infections, using cut-off points at 7, 14, 21 and 28 days. There was no significant difference at any of these time-points $(p=N S)$. Conclusions The pleural infection rate with an IPC was slightly higher than previous published series. There was however no significant increase in pleural infection in those receiving chemotherapy, although these analyses did not account for variability in tumour type, tumour stage, performance, co-morbidity and leucopenia at time of insertion. While the analyses were not significant, this needs to be retested in larger or combined registries of patients treated with IPCs for pleural malignancy.

Abstract S116 Table 1 A 2x2 contingency table assessing numbers of pleural infection with systemic chemotherapy in patients with an IPC

\begin{tabular}{llll}
\hline & Pleural infection & No pleural infection & \\
\hline Chemo & 4 & 11 & 15 \\
No chemo & 7 & 78 & 85 \\
& 11 & 89 & \\
\hline
\end{tabular}

